\title{
CHARLES DIDIER, PASSEUR CULTUREL ENTRE FRANCE ET ITALIE A L'EPOQUE ROMANTIQUE
}

\begin{abstract}
L'articolo propone la ricostruzione culturale della figura dello scrittore elvetico di lingua francese Charles Didier (1805-1864), la cui opera e la cui figura vengono ricontestualizzate rispetto ai suoi rapporti con l'Italia e, in particolar modo, nel suo ruolo di primo traduttore francofono dei Canti di Leopardi.

Parole chiave: Charles Didier, Giacomo Leopardi, Rapporti culturali franco-italiani, Transfer letterario e culturale, Ricezione dell'Italia nella cultura francese, Romanticismo europeo.
\end{abstract}

Charles Didier est un écrivain suisse d'expression française, né à Genève le 15 septembre 1805. Il se destine rapidement à la littérature, mais, sans fortune personnelle, entre en 1825 chez Charles-Victor de Bonstetten pour s'occuper d'un des petit-fils de celui-ci. La rencontre avec ce penseur ami de Mme de Staël va orienter toute sa vie. Bonstetten, qui a séjourné trois fois en Italie, a écrit, outre une abondante correspondance, plusieurs ouvrages dont Voyage sur la scène des six derniers livres de l'Enéide, suivi de quelques observations sur le Latium moderne, daté de 1805, mais publié dès l'automne 1804. Or, l'Italie va compter plus que tout dans la vie de Charles Didier, et s'il est passé à la postérité, c'est par les livres et les articles qu'il a laissés sur ce pays alors en profonde mutation.

Il effectue un très long voyage en Italie (1827-1830), parfois accompagné par un ami genevois, David Richard, quand il visite le nord du pays. Au début de son voyage, en septembre 1827, il s'installe d'abord à Florence et sympathise, notamment, avec Giovan Pietro Vieusseux ${ }^{1}$, qui tient un célèbre

*sophie.guermes@univ-brest.fr

${ }^{1}$ Charles Didier (1831a: 17) écrit au sujet du gabinetto et de son directeur: «On y lit tous les journaux, quelle que soit leur couleur, les livres nouveaux, les recueils scientifiques 
gabinetto et dirige l'Antologia, et avec qui il correspondra jusqu'à ce qu'il devienne aveugle, au début des années 1860; il devient aussi l'ami d'Hortense Allart, femme de lettres qui vivait à cette époque en Italie, ainsi que de Lamartine et de Gino Capponi ${ }^{2}$. Puis, il séjourne longuement à Rome, et sillonne le pays du nord au sud. Au sud, il ne s'est pas contenté de Naples et de la Sicile: il a voulu tout voir. Et il est particulièrement intéressé par la vie de ses contemporains, dans les Pouilles, les Abruzzes ou encore en Calabre. Il va rapporter d'Italie la matière de plusieurs articles publiés à partir de 1831 dans la Revue encyclopédique et la Revue des Deux Mondes (dont la première étude en langue française sur Leopardi), les lettres qui composeront le volume Campagne de Rome (1842), et un roman, Rome souterraine (1833). De retour en France, il rencontre à Genève en octobre 1830, l'historien et économiste de Sismondi (1773-1842), qui avait accompagné Mme de Staël en Italie en 1808, et dont il avait lu la volumineuse Histoire des républiques italiennes au Moyen Âge. Il rencontre aussi Frédéric Lullin de Châteauvieux, auteur de Lettres écrites en Italie en 1812 et 1813.

Son premier article a un titre bien différent de ce qu'on attendrait d'un récit de voyage: Coup d'œil sur la statistique morale et politique de l'Italie $e^{3}$. Signe de la résonance des propos de l'auteur, et de l'efficacité de son réseau amical, ces pages sont aussitôt traduites en italien et paraissent en septembre sous forme d'une brochure.

L'objectif de Charles Didier est de témoigner. C'est ce qui le différencie des voyageurs du Grand Tour qui rapportent des impressions poétiques et artistiques. Il définira clairement ses objectifs dans son deuxième article, Notice sur le royaume des Deux-Siciles: "Assez de voyageurs ont cherché en Italie des tableaux et des pierres, j'y ai cherché des institutions et des hommes» (Didier 1831b: 438); mais on comprend son but dès la lecture du premier article. Charles Didier est très imprégné de culture italienne, ses textes de fiction le montrent suffisamment, mais dans ses articles, il travaille en journaliste: il s'agit de reportages fondés sur une enquête approfondie, même s'il a l'honnêteté de souligner que les chiffres qu'il avance ne sont pas absolument exacts:

et littéraires, et les brochures. Cet avantage, inappréciable au-delà des Alpes, suffirait pour mériter à Florence le nom d'oasis de l'Italie. Le directeur de ce cabinet l'est en même temps de l'Anthologie, le meilleur recueil littéraire de l'Italie, et le plus indépendant».

${ }^{2}$ Voir Charles Didier, Lettere a Gian Pietro Viesseux e a Gino Capponi, Carteggio Viesseux, mss. cassetta 33, Biblioteca nazionale centrale di Firenze.

${ }^{3}$ Il paraît dans la Revue encyclopédique, tome 49, en deux parties, en janvier (pp. 24-39) puis février (pp. 288-301) 1831, puis en brochure séparée, la même année (Didier 1831a). 
L'Italie compte une population de vingt-et-un millions d'habitants environ, répandus sur une surface de 90,652 milles carrés; ce qui fait un peu plus de 231 habitants par mille carrés [...]. Son armée active [est] de 116,941 hommes. Tels sont les chiffres recueillis à grand peine pendant un voyage minutieux de plusieurs années. Nous ne les donnons ici que comme une probabilité, une approximation; car les études statistiques sont si négligées en Italie, et si entravées par les gouvernements, qu'il n'est guère possible de présenter des résultats sûrs. Rien de moins certain, par exemple, que la population du royaume des Deux-Siciles, et les revenus des États de l'Église. Nous avons été en rapport, à ce sujet, avec les autorités elles-mêmes, et plusieurs nous ont avoué leurs doutes et leur ignorance (Didier 1831a: 5).

Mais ce qui l'intéresse ne se borne pas à la transmission de statistiques: il veut attirer l'attention sur ce que fut l'Italie, et ce qu'elle est devenue. En effet, c'est le passé glorieux du pays qui inspire les patriotes, dont Charles Didier se fera l'écho aussi bien dans ses articles que dans ses livres:

Voilà donc vingt-et-un millions d'hommes pauvres, sur la terre la plus fertile, sous le ciel le plus doux d'Europe: perdant en questions futiles de grandes facultés intellectuelles; sans industrie au milieu de tous les produits de la nature; sans commerce avec une position géographique admirable; sans existence politique, enfin, avec tous les éléments et toutes les traditions de destinées brillantes. Et si nous ouvrons leurs annales, qu'y voyons-nous? Un peuple jadis puissant et fort [...]. Où trouver les causes d'une pareille décadence ? - Dans une combinaison de circonstances longtemps malheureuses, dans l'ineptie constante des gouvernements, dans sa gloire même qui lui a valu d'être le foyer de l'Église romaine, le centre du monde ecclésiastique (Didier 1831a: 5).

Il considère que le royaume de Sardaigne et la Lombardie seront les deux acteurs majeurs d'un changement de destin pour le pays. La Toscane et Bologne ont aussi accueilli avec espoir l'annonce de la révolution de 1830 à Paris. Outre la domination autrichienne, le poids de l'influence du clergé bride dans de nombreuses régions les velléités émancipatrices. Très important dans les États pontificaux, ce poids atteint évidemment son paroxysme à Rome:

L'organisation papale est une sorte de noli me tangere. On ne sait pas où y toucher: il semble un squelette qui doit tomber en poussière au grand air. Si la Toscane administrative nous a paru un labyrinthe, quel nom donner à l'administration romaine? C'est un chaos d'institutions hétérogènes qui se combattent comme les éléments avant la création. De cette lutte même naît un certain équilibre; car enfin la machine va (Didier 1831a: 18).

La complexité de ses rouages, la puissance du Saint-Office, la peur des Autrichiens composent un alliage qui encourage l'immobilisme. Charles Didier va écrire un roman, Rome souterraine, où des Romains et des 
habitants du Latium, tous carbonari, s'allient à l'occasion d'un conclave pour lutter contre les Autrichiens et faire élire un pape républicain. Ils vont doublement échouer. Cet échec illustre l'opinion émise par Didier dans son premier article: «On a parlé récemment d'une révolution à Rome. Nous n'y avons pas cru; nous la verrions de nos yeux que nous n'y croirions pas encore. Rome voudrait faire un pas, qu'elle ne le pourrait point. Elle est comme Gulliver garrotté par les pygmées [...]. Il faudrait une combinaison que nous ne prévoyons pas, pour qu'une révolution partît de Rome» (Didier 1831a: 18-19). Et l'analyse de l'écrivain est prémonitoire, car Rome sera jusqu'en 1870 l'obstacle à l'unité du pays. Cela n'empêche pas Didier d'aimer profondément la future capitale italienne:

Quelle existence miraculeuse que celle de cette ville si radieuse encore au milieu de ses splendeurs éteintes, si forte dans sa faiblesse, si imposante dans son isolement! [...] Nous désirons sans doute l'émancipation de ses provinces; mais [...] nous voulons Rome, telle que cinquante générations l'ont faite; Rome avec ses ruines, avec ses arts, Rome avec sa pourpre pontificale, Rome avec son désert (Didier 1831a: 293-294).

Son deuxième article (Didier 1831b), «Notice sur le royaume des DeuxSiciles», paraît six mois plus tard, toujours dans la Revue encyclopédique. Il y dénonce la misère qui frappe les régions du sud, l'obscurantisme du clergé, les tortures infligées aux carbonari.

En janvier 1832 parait, dans la même revue, un autre article important, dont l'orientation politique est inscrite dès le titre: Les Trois principes. Rome, Vienne, Paris. Il voit dans la paralysie durable de l'Italie la résurgence de l'ancienne opposition entre les guelfes (partisans du pape) et les gibelins (partisans de l'empereur). Il estime que si la papauté s'est affaiblie au fil des siècles, c'est à la fois «qu'elle a déserté la cause des peuples pour embrasser celle des princes» (Didier 1832: 40), et qu'elle a refusé tout aggiornamento - il n'emploie pas cette expression, mais en exprime l'idée: l'écart entre la papauté et le monde moderne est un leitmotiv de la littérature française du XIX ${ }^{\mathrm{e}}$ siècle. On le trouve même chez écrivains catholiques, comme Lamennais (qui fut pendant plusieurs années un ami de Charles Didier, avant de se brouiller avec lui) ${ }^{4}$. Mais Didier est l'un des premiers à l'affirmer: «le catholicisme a fait son temps, comme institution politique» (Didier 1832: $41)^{5}$. Il n'est pas opposé au pape, mais rêve d'un pape social, qui ressusciterait le troisième principe, le principe populaire, dont la France a rappelé

${ }^{4}$ Ce sont précisément des écrivains et penseurs catholiques qui ont amené Jean XXIII à décider la tenue du concile Vatican II.

${ }^{5} \mathrm{Cf}$. aussi «et c'est à ce fantôme impuissant, réclamé du sépulcre, qu'on veut lier un siècle jeune et bouillant, qui a soif d'avenir et de nouveauté» (Didier 1832: 54). 
avec la Révolution de 1789 puis celle, plus récente et plus brève, de 1830, qu'il pouvait resurgir; mais, dans l'état actuel des choses (sur le trône de saint Pierre se succèdent des papes issus des zelanti, la partie la plus conservatrice de la Curie: Grégoire XVI, qui excommunia Lamennais, en est le dernier exemple, et son pontificat dure longtemps, de 1831 à 1846), pour devenir indépendante et une, l'Italie devra s'affranchir de la double tutelle pontificale et autrichienne, en s'inspirant des révolutionnaires parisiens: «c'est la France qui tient le fil des destinées de la Péninsule italienne» (Didier 1832: 63). Les Italiens doivent devenir des «prêtres de l'avenir» (Didier 1832: 61), et pour cela acquérir suffisamment d'esprit critique. Charles Didier, prônant l'exemple français, rappelle que lui-même est Suisse et s'exprime donc objectivement. Et il fait preuve une fois encore d'une conscience aiguë de la solidarité entre les peuples européens: les problèmes politiques et sociaux de ceux-ci traversent les frontières, ils ne représentent plus des cas isolés. De nouveau, ce long texte engagé fut traduit et parut sous la forme d'une brochure de cinquante-huit pages en Italie.

Didier présente et analyse dans ses articles la situation de l'Italie contemporaine; il n'évoque généralement pas les artistes ou les écrivains; mais il fait une exception, en présentant un auteur encore inconnu des Français: Leopardi. Il publie sur ce poète dans la Revue encyclopédique, en janvier 1833, un article signé SR, on ignore pourquoi; puis un nouvel article dans La Paix, en 1837.

Les «Canti del conte Giacomo Leopardi» ont été publiés en 1831 à Florence chez Piatti. Didier commence son article en citant des vers, qu'il traduit lui-même, dans lesquels Leopardi déplore l'état de sa patrie. Voici l'original et la traduction:

O patria mia, vedo le mura e gli archi

E le colonne e i simulacri e l'erme

Torri degli avi nostri,

Ma la gloria non vedo,

Non vedo il lauro e il ferro ond'eran carchi

I nostri padri antichi. Or fatta inerme,

Nuda la fronte e nudo il petto mostri.

Oimè quante ferite,

Che lividor, che sangue! oh qual ti veggio,

Formosissima donna! Io chiedo al cielo

E al mondo: dite dite;

Chi la ridusse a tale? [...]

(All'Italia, vv. 1-12)
Ô ma patrie, je vois les murs, les arcs, les colonnes, les statues, les tours solitaires de nos ancêtres; mais la gloire, je ne la vois pas; je ne vois pas le laurier et le fer qui chargeaient nos pères. Maintenant désarmée, tu ne montres plus qu'un front nu, qu'une poitrine nue. Hélas! que de blessures! quelle pâleur! que de sang! En quel état te vois-je! ô femme aux divines formes! Je crie au ciel et au monde: Dites, oh! dites-moi qui l'a pu réduire ainsi? 
Di noi serbate, o gloriosi, ancora

Qualche speranza? in tutto

Non siam periti? A voi forse il futuro

Conoscer non si toglie. Io son distrutto

Nè schermo alcuno ho dal dolor, che scuro

M'è l'avvenire, e tutto quanto io scerno

E' tal che sogno e fola

Fa parer la speranza. [...]

(Ad Angelo Mai, vv. 31-38)
Gardez-vous sur nous, hommes glorieux du passé, gardez-vous encore quelque espoir; et ne sommes-nous pas morts tout entiers? L'avenir, sans doute, n'est pas pour vous un mystère; et détruit, anéanti par la douleur, l'avenir n'est, hélas! pour moi qu'obscurité, et tout ce que je vois est tel que l'espérance ne me semble qu'un songe et qu'une chimère (Didier 1833: 171) ${ }^{6}$.

Fragments pris «au hasard», écrit l'auteur de l'article. En fait, le choix de commencer celui-ci par la déploration de la gloire perdue est très significatif (à la fin de son article, Charles Didier évoque aussi la nature et l'amour, mais développe moins ces aspects). L'écrivain suisse a lui-même été, on vient de le voir, très sensible à la situation de l'Italie au moment où il s'y était rendu; s'étonnant qu'on ait laissé sortir le livre de Leopardi, il pense que s'il avait été en prose il aurait été censuré.

Il y a donc une communauté de vues entre les deux écrivains, pour ce qui concerne la situation politique du pays. Ce qui plaît encore à Didier, c'est la tristesse de Leopardi, qu'il éprouve lui-même, comme il l'écrira à Marie d'Agoult: «Vous me demandez [...] si je suis triste. Vous savez bien que la tristesse est mon état normal, et ma vie pour ainsi dire. Je ne suis pas triste pour telle ou telle raison, je suis triste parce que je suis triste» ${ }^{7}$. Et il ajoute que même s'il obtenait tout ce qu'il désirait il le resterait. La tristesse de Leopardi est accrue par l'état de l'Italie; mais elle lui est d'abord consubstantielle, et c'est évidemment un trait que son commentateur sait reconnaître: «C'est un homme fondamentalement triste. Qu'il jette les yeux sur l'Italie, il gémit; qu'il se retire en lui-même, il gémit encore. La vie n'a point d'asile où les pleurs se changent en joies pour lui» (Didier 1833: 172).

En revanche, malgré son caractère chagrin, Charles Didier espère que la situation changera et que les patriotes vaincront; Leopardi, lui, n'a pas cette espérance; il se réfugie, selon Didier ${ }^{8}$, dans le rêve nostalgique d'une vie sauvage dont l'écrivain suisse dénonce au contraire l'inhumanité. C'est le seul point de désaccord entre eux.

${ }^{6}$ Il s'excuse pour finir «de l'avoir si mal traduit» (Didier 1833: 176).

${ }^{7}$ Lettre à Marie d'Agoult, 17 septembre 1837, Bibliothèque nationale de France, NAF 25186, f. 290.

${ }^{8}$ «Nous ne croyons pas, nous, que le monde dorme; jamais sa veille au contraire ne fut plus ardente», écrit Didier. «Nous ne croyons pas non plus à ce printemps de la terre que chante le poète, et moins encore à ce bonheur idéal des forêts de Californie qu'il préconise, et où nous ne voyons que violence, ignorance et brutalité. Cette vie sauvage si longtemps vantée est misérable; l'auteur lui-même n'en voudrait pas, mais il faut pardonner beaucoup à une douleur italienne» (Didier 1833: 173). 
Outre son sentiment patriotique, ce qui a plu à Charles Didier, c'est l'originalité de Leopardi, qui a surpris les Italiens:

Faite depuis si longtemps à une poésie de convention et de réminiscences classiques, poésie froide et morte, l'Italie s'est étonnée de l'individualité empreinte dans les chants du comte L. [...]. Plus d'un lui a fait un crime d'avoir quitté le grand chemin banal de la routine pour aller puiser à des sources intimes. Quant à moi, c'est par ces crimes mêmes qu'il m'a plu, qu'il m'a touché, et je tiens à honneur de révéler le premier à la France l'existence d'un poète original dont le nom, encore inconnu de ce côté des Alpes, grandit au-delà (Didier 1833: 175) .

Parallèlement à ses publications régulières dans la Revue encyclopédique, Didier collabore aussi en 1831 à la Revue des Deux Mondes, où il publie le récit d'un épisode dramatique survenu en 1828 pendant l'insurrection du Cilento, Les Capozzolli et la police napolitaine (Didier 1831c): il s'agissait de l'exécution de trois frères qui avaient fait partie d'une révolte réprimée à Salerne avec le concours de l'ambassadeur de France à Naples, le duc de Blacas; il publie aussi dans d'autres volumes de la même revue des Souvenirs de Calabre (Didier 1831d) ${ }^{10}$.

Il rassemble également des notes en vue de l'écriture d'un roman. Son journal ${ }^{11}$ nous apprend que c'est le 18 septembre 1832 qu'il commence la rédaction de Rome souterraine. Il écrit aussi les cinq longues «lettres» (en fait, des articles dédiés à des écrivains ou artistes qu'il fréquentait alors) qui paraissent séparément dans la Revue encyclopédique et seront reprises dix ans plus tard dans le recueil Campagne de Rome. La première s'intitule De quelques paysages italiens. La campagne de Paris et la campagne de Rome; la deuxième lettre, Les Maremmes, paraît dans la même revue, en janvier 1833. À la fin de l'année, Rome souterraine est publié, en deux volumes, à la Librairie de la Revue encyclopédique. Mais c'est la Revue de Paris qui avait annoncé le roman en publiant, en avant-première, le chapitre $U n$ conclave, précédé d'une note élogieuse ${ }^{12}$.

${ }^{9}$ Sur la présentation de Leopardi par Charles Didier, voir Bellucci (1996: 358-359, $364,420)$.

${ }^{10}$ L'article consacré aux «Grecs de Calabre» est loin d'être un simple reportage. Didier y écrit à la fin: «J'appelle de tous mes vœux la résurrection de l'Italie, et cherchant, pour un présent dont j'ai vu les souffrances, des consolations dans un passé dont les gloires vont au cœur et réchauffent la pensée, j'y trouve pour l'avenir que j'invoque, que nous invoquons tous, des espérances et des garanties» (Didier 1831d: 116).

${ }^{11}$ Non publié, et incomplet, ce journal rédigé entre 1826 et 1848 est conservé à la Bibliothèque Mazarine (Ms Lov. D672 bis et ter).

12 «[ROME SOUTERRAINE. Tel est le titre d'un roman de M. Charles Didier, qui paraîtra dans la première quinzaine d'octobre. Connu pour ses appréciations pratiques et pittoresques de l'Italie, l'auteur caractérise d'une manière originale et hardie, dans ce nou- 
Le roman a connu huit éditions, dont les parutions s'étirent sur une quinzaine d'années, et une contrefaçon belge. Il a donc remporté un grand succès, et on comprend pourquoi: toute la partie romanesque pouvait divertir en même temps qu'émouvoir. Il y avait un héros modéré, Anselme, et un patriote passionné, Marius, ainsi que de multiples portraits de carbonari; une histoire d'amour; de l'espionnage; de l'humour; un personnage de prêtre hors du commun, le cardinal de Pétralie, Grand-Pénitencier; la restitution de la vie quotidienne dans plusieurs quartiers de Rome, en particulier le Trastevere; mais l'arrière-plan politique, retraçant l'histoire immédiatement contemporaine (en particulier le conclave de 1823), instruisait également les lecteurs - lecteurs français, mais aussi italiens (il y eut sept traductions italiennes, de 1845 à 1879), anglais (deux traductions, en 1843 et 1860) et allemands (également deux traductions, l'une dès 1835, l'autre en 1846).

Par la suite, Charles Didier a encore été inspiré par l'Italie, qu'il s'agisse ou non de textes de fiction. Il a fait paraître en 1844 un recueil de nouvelles, Raccolta. Mours siciliennes et calabraises; en 1845 un roman historique, Caroline en Sicile, qui relate l'exil du dernier roi, Ferdinand, et de sa femme Caroline, après la prise de pouvoir de Joseph Bonaparte sur le royaume de Naples; en 1859 un autre recueil de nouvelles, Les Amours d'Italie, dont la structure s'inspire de celle du Décameron. Il a également édité, en 1842, des Chants populaires de la campagne de Rome; il a aussi prolongé ses articles d'histoire et de politique contemporaines par deux brochures: 1'Introduction à L'Italie politique et ses rapports avec la France et l'Angleterre, du général Pepe, en 1839; et, dix ans plus tard, Question sicilienne.

vel ouvrage, la vie interne et pour ainsi dire occulte de la 'Niobé des nations'. Il y a deux Italie, l'Italie visible et l'Italie invisible. La première est celle des églises, des palais, des ruines et des tableaux; elle est accessible à tous les passants, elle est dans tous les livres. L'autre est l'Italie des sectes, l'Italie souterraine, et celle-là n'est pas écrite: mystérieuse et muette, elle ne se révèle qu'à quelques adeptes patiens qui se dévouent à descendre dans ces cryptes ténébreuses. Voilà l'Italie de M. Didier, et il a été la chercher, celle-là, comme il le dit lui-même, village à village, homme à homme. C'est en puisant plusieurs années à ces sources cachées qu'il a réussi à être neuf sur un sujet si vieux. Son livre est une véritable initiation. Il nous fait voir des hommes et d'ardentes passions sociales où les autres ne voient que des morts et des tombeaux. La scène se passe à Rome et dans sa grande et austère campagne, à la fin de la restauration; mais l'Italie tout entière concourt à l'action. Une fable fort simple en lie les diverses parties. Les carbonari avec leurs conspirations, les sanfédistes et leurs intrigues jouent un rôle dans le drame. Nous pouvons dès ce moment prédire au roman de M. Didier un grand succès. Les tristes événemens sardes lui assurent d'ailleurs un intérêt tout actuel. Le fragment suivant donnera une idée de sa manière. C'est la description d'après nature du conclave et de la grande neuvaine qui suit la mort du pape.]. - [N. du D.]» (Note 1933: 5). L'extrait du roman suit aux pages 5-13. 
À la fin du roman Caroline en Sicile, Charles Didier abandonne la fiction pour reprendre son rôle d'analyste historique, et porte un jugement sévère sur les Anglais, mais aussi sur le roi Ferdinand:

à la chute de Napoléon, l'Angleterre abandonna la Sicile avec une perfidie insigne; et, au mépris des promesses les plus solennelles, les plus saintes, elle livra ses partisans, tous ceux quis 'étaient compromis par elle et pour elle, aux vengeances d'une cour implacable [...]. Rentré en possession de son trône, le vieil exilé de la Ficuzza foula aux pieds, lui aussi, toutes ses promesses et viola sans scrupule tous ses serments. Il est vrai que pour décharger sa conscience timorée du crime de parjure, il inventa, ou l'on inventa pour lui un tour ingénieux. Comme roi de Sicile, il était Ferdinand III, et Ferdinand IV comme roi de Naples; il réunit en un seul les deux royaumes et prit le titre de Ferdinand $1^{\mathrm{er}}$, roi des Deux-Siciles; le tour fait, il déclara avec un aplomb sublime qu'un nouveau règne commençait, et que le Ferdinand numéro un ignorait les engagements qu'avaient pu prendre les Ferdinand trois et quatre (Didier 1845: 321-322) ${ }^{13}$.

Marie-Caroline d'Autriche, sœur de Marie-Antoinette, avait épousé Ferdinand, Bourbon d'Espagne, cousin de Louis XVI devenu aussi son beau-frère, roi faible qu'elle domina politiquement autant qu'elle le put. Ils vivaient à Naples mais la campagne d'Italie menée par Bonaparte puis la proclamation de la République parthénopéenne ${ }^{14}$ les obligèrent à s'exiler en Sicile, sous domination anglaise (les Anglais soutenaient les Bourbons contre Bonaparte); elle fit échouer la révolution républicaine en Sicile et revint à Naples. Quand Napoléon $1^{\text {er }}$ plaça son frère Joseph sur le trône de Naples, elle fut contrainte de repartir en Sicile, d'où elle fut chassée par les Anglais en 1813, un an avant sa mort à Vienne.

À partir de 1820, en Sicile comme sur la péninsule italienne, des insurrections se produisirent contre la domination étrangère. Ayant pour point de départ l'abolition, par Ferdinand, de la constitution de 1812, elles culminèrent en 1848, année de multiples révolutions en Europe; l'un des fils de Ferdinand et de Marie-Caroline régnait depuis 1830; à l'issue de multiples conflits avec son peuple, il mourut en 1859; son fils eut un

${ }^{13} \mathrm{Cf}$. aussi Didier (1845: 210), où Ferdinand était présenté comme un «cœur faible, irrésolu», un «mannequin royal» qui obéissait «docilement au mouvement imprimé par» sa femme.

${ }^{14}$ Voir à ce sujet le roman d'Alexandre Dumas La San Felice (1864), qui retrace sous forme fictionnelle des événements historiques: l'établissement de la république par des patriotes inspirés par la Révolution française et l'institution de la Première République en France, et la répression sanglante menée par le cardinal Ruffo avec l'aide des Anglais. Dumas, qui avait vécu à Naples de 1861 à 1864, était très apprécié des partisans du Risorgimento. Il fut l'ami de Garibaldi, qui le nomma directeur des fouilles et des musées de la ville. 
règne éphémère puisque la Sicile fut annexée au Royaume d'Italie: il dut capituler en février $1861^{15}$. En 1849, lorsque Charles Didier fait paraître la brochure intitulée Question sicilienne, le sort de l'île est donc incertain, et les Français ne comprennent pas les événements qui s'y déroulent, faute de bien connaître les données du problème; il espère en présentant ceux-ci rendre plus clairs les enjeux de la lutte. Les Bourbons n'ont pas respecté les droits des Siciliens: ils ont porté atteinte à la constitution, levé de nouveaux impôts sans consulter le Parlement, et fait arrêter tous ceux qui s'opposaient à eux. C'est la raison pour laquelle les Anglais avaient eu un prétexte pour chasser Ferdinand. Après le congrès de Vienne et le retour de celui-ci, devenu roi des Deux-Siciles, «titre passablement ridicule» selon Charles Didier, la Sicile ne veut pas être absorbée par Naples et devenir «une simple province napolitaine» (Didier 1849: 16). Une trentaine d'années plus tard, la question se pose cette fois d'un rattachement de la Sicile à l'Italie alors en lente voie de constitution. Entre-temps, on l'a vu, Charles Didier avait visité l'île sous l'occupation autrichienne et gardait le souvenir d'une «colonie exploitée par la métropole», Naples pillant ses richesses sans la moderniser, et réprimant avec dureté les tentatives de soulèvement. La situation aboutit à des demandes répétées de réformes, qui restèrent lettre morte. L'insurrection de Palerme, qui commença le 12 janvier 1848, déboucha sur l'élaboration d'une nouvelle constitution. Ruggero Settimo ${ }^{16}$ puis Pietro Lanza devinrent présidents du Conseil; mais l'île demeurait une province de Naples. Le roi avait institué par le décret du 6 mars un ministère pour les affaires de la Sicile, ce qui était en soi une absurdité et, de fait, fut considéré par les Siciliens comme non conforme à la constitution. Le nouveau Parlement voulut réformer l'île et mettre fin aux abus: «C'était entreprendre les travaux d'Hercule; les étables d'Augias étaient propres, comparées à l'administration napolitaine en Sicile. [...] Le cri de Fuori Borboni! retentit d'un bout à l'autre de l'île» (Didier 1849: 35, 37). Mais le roi avait conservé de nombreux soldats; l'annonce de sa destitution par Ruggero Settimo provoqua des représailles sanglantes, aboutissant au sac de Messine sur lequel Charles Didier s'arrête longuement.

L'annonce de la chute de Louis-Philippe, hostile aux Siciliens (il était cousin des Bourbons et fils d'une princesse Bourbon de sang non royal) ${ }^{17}$, fut bien accueillie par ceux-ci, qui espérèrent le soutien de la nouvelle République française; mais celle-ci ne reconnut pas la Sicile, et c'est pour tenter

${ }^{15}$ Ces événements sont évoqués dans le roman de Giuseppe Tomasi di Lampedusa Il Gattopardo publié en 1958 après la mort de son auteur.

${ }^{16}$ «l'un des hommes les plus considérés de toute la Sicile» (Didier 1849: 34).

${ }^{17}$ Sa mère avait pour arrière-grand-père l'un des enfants que Louis XIV avait eus avec Mme de Montespan. 
de faire évoluer la situation que Didier écrivit sa brochure: il y appelait la Deuxième République à l'aide, mais ne fut pas entendu, Louis-Napoléon Bonaparte ayant commencé à mener vis-à-vis de l'Italie une politique ambiguë qu'il poursuivit une fois devenu l'empereur Napoléon III ${ }^{18}$.

De nombreux écrivains français ont soutenu les combats des patriotes italiens pour l'unité et l'indépendance du pays. Charles Didier a été le premier à les faire connaître en France et il a aussi été le passeur le plus complet: en effet, il a dans ses articles comme dans ses fictions donné des renseignements exacts sur la situation morale et politique du pays de la fin des années 1820 à la fin des années 1840; il a aussi rappelé le glorieux passé de celui-ci, des Gracques à Dante en passant par quelques grands papes; et il a eu de l'avenir une vision juste. Ni Hugo, ni Lamartine, ni George Sand ni même Edgar Quinet (pourtant grand connaisseur de l'Italie et de ses «révolutions») n'ont livré un panorama et une analyse aussi exhaustifs de l'état du pays à un moment clef de son histoire. Les Italiens ne s'y sont d'ailleurs pas trompés: on le fit membre de l'Académie d'Arezzo, en raison de Rome souterraine (Journal, 31 octobre 1839). Il note encore dans son Journal le 14 mai 1844: "Visite d'un jeune Milanais, qui vient spontanément m'exprimer, en son nom et au nom de sa famille, leur sympathie pour Rome souterraine qui est, dit-il, le bréviaire de la jeunesse italienne»; et enfin le 14 mars 1849: «Pescatori, envoyé de la République romaine, me dit qu'elle va me déclarer citoyen romain à cause de Rome souterraine». On voit bien aussi que son roman de 1833 était encore considéré, seize ans plus tard, comme porteur d'une vision juste. Charles Didier méritait donc d'être tiré de l'oubli pour ces raisons, et plusieurs chercheurs, ces dernières années, ont contribué à rééditer certains de ses livres ${ }^{19}$.

${ }^{18}$ Mais sans doute Charles Didier lui-même ne croyait plus en un soutien, quand il rédigea le post-scriptum dans lequel il annonçait la reprise de la lutte armée; il émit alors le souhait que la Sicile se constitue sans secours extérieur, de façon à être plus libre.

${ }^{19}$ À une exception près, ces chercheurs sont Italiens, alors que les deux principaux commentateurs de Charles Didier, à la fin du XIX ${ }^{\mathrm{e}}$ siècle et dans la première partie du $\mathrm{XX}^{\mathrm{e}}$ siècle, avaient été, pour l'un, Suisse (Marc Monnier), pour l'autre, Américain (John Armstrong Sellards, qui était professeur à l'université de Stanford): Didier (1989, 1991, 1994, 1996, 2007, 2008); Didier \& Galzerano (2003); Calì (1996). 


\section{BIBLIOGRAPHIE}

Bellucci, N. (1996). Leopardi e i contemporanei. Testimonianze dall'Italia e dall'Europa in vita e in morte del poeta. Firenze: Ponte alle Grazie.

Calì F. (a cura di). (1996). La Sicilia di Charles Didier. Sogno e incanto di un viaggiatore romantico. Acireale: Bonanno.

Didier, Ch. (1831a). Coup d'œil sur la statistique morale et politique de l'Italie. Paris: Bureau de la Revue Encyclopédique.

Didier, Ch. (1831b). Notice sur le royaume des Deux-Siciles. Revue encyclopédique, L [avril], 438-470.

Didier, Ch. (1831c). Les Capozzolli et la police napolitaine. Revue des Deux-Mondes, II, 58-69.

Didier, Ch. (1831d). Souvenirs de Calabre. Revue des Deux-Mondes, III, 93-116.

Didier, Ch. (1832). Les Trois principes. Rome, Vienne, Paris. Revue encyclopédique, LIII [janvier-mars], 37-66.

Didier, Ch. (1833). Canti del conte Giacomo Leopardi - Chants du Comte Jacques Leopardi. Florence, Piatti. Revue encyclopédique, 57 [janvier], 171-176.

Didier, Ch. (1845). Caroline en Sicile. Paris: Jules Labitte [t. IV].

Didier, Ch. (1849). Question sicilienne. Paris: Michel Lévy frères.

Didier, Ch. (1989). La Sicilia pittoresca, traduzione di R. Volpes, introduzione di M.C. Ruggieri Tricoli. Palermo: La Ginestra.

Didier, Ch. (1991). La questione siciliana, a cura di G. Falcetta. Palermo: Edizioni Novecento.

Didier, Ch. (1994). Campagna romana, a cura di R. Cincotta. Viterbo: Union Printing.

Didier, Ch. (1996). Voyage en Italie, préf. d'A. Brudo. Genève: Slatkine.

Didier, Ch. \& Galzerano G. (2003). I Capozzoli e la rivolta del Cilento del 1828. Casalvelino Scalo: Galzerano.

Didier, Ch. (2007), Rome souterraine, éd. critique par S. Guermès. Genève: Droz.

Didier, Ch. (2008). Viaggio in Calabria, introd. e trad. di S. Napolitano. Soveria Mannelli: Rubbettino.

Note. (1833). La Revue de Paris, 55. 


\section{CHARLES DIDIER, PASSEUR CULTUREL BETWEEN FRANCE AND ITALY IN THE ROMANTIC PERIOD}

\section{Summary}

The article offers the cultural reconstruction of the Swiss French-writing author Charles Didier (1805-1864), whose work and figure are put into the context of his relationships with Italy; in particular, he is presented as the first French-speaking translator of Leopardi's Canti.

Keywords: Charles Didier, Giacomo Leopardi, Cultural Relationships between France and Italy, Literary and Cultural Transfer, Italian reception in French Culture, European Romanticism. 